\title{
LA INDAGACIÓN GUIADA COMO ESTRATEGIA METODOLÓGICA PARA EL DESARROLLO DE COMPETENCIAS CIENTÍFICAS EN ESTUDIANTES DE EDUCACIÓN MEDIA
}

\author{
Clara Inés Ortiz Viviescas \\ Universidad Internacional Iberoamericana (México) \\ clarainesortizv@hotmail.com·https://orcid.org/0000-0002-0724-1478 \\ Magdalena Suárez-Ortega \\ Universidad de Sevilla (España) \\ msuarez@us.es $\cdot \underline{\text { https://orcid.org/0000-0002-0188-3074 }}$
}

\begin{abstract}
Resumen. Los avances tecnológicos requieren del desarrollo del pensamiento científico con el cual los estudiantes puedan acceder y transformar su entorno. Esto propende por la enseñanza de una ciencia actualizada, contextualizada y motivadora que despierte el interés en los estudiantes y que privilegie el desarrollo de competencias científicas. Bajo esta premisa, el objetivo del presente artículo es presentar los resultados obtenidos al implementar una estrategia metodológica basada en la indagación guiada, en la adecuación de prácticas de laboratorio de física. La estrategia se implementó en tres colegios de Bogotá (Colombia), en una muestra conformada por 145 estudiantes de educación media, efectuando el seguimiento de las interacciones de los estudiantes con el modelo de prácticas de laboratorio propuesto, y analizando los progresos de sus desempeños inherentes a las competencias sugeridas en la investigación. Desde una metodología mixta, de corte descriptivo analítico, se implementaron pruebas de entrada y salida diseñadas ad hoc, que establecieron el nivel de apropiación de las competencias, antes y después de la implementación de la estrategia metodológica. Los resultados obtenidos dan cuenta de la efectividad de la estrategia al interior de los contextos educativos considerados. Se concluye que la estrategia presenta un carácter innovador y relevante, al proponer elementos orientadores y didácticos para la enseñanza de las ciencias, acercando a los estudiantes hacia los nuevos desarrollos tecnológicos y científicos actuales.
\end{abstract}

Palabras clave: indagación guiada, estrategia metodológica, competencias científicas, prácticas de laboratorio, educación media. 


\title{
THE GUIDED INDAGATION AS A METHODOLOGICAL STRATEGY FOR THE DEVELOPMENT OF SCIENTIFIC COMPETENCES IN MIDDLE EDUCATION STUDENTS
}

\begin{abstract}
Technological advances require the development of scientific thinking in which students can access and transform their environment. This tends to teach an updated, contextualized and motivating science which awakens interest in students and that emphasizes in the development of scientific competences. Under this premise, the objective of this article is to present the results obtained when implementing a methodological strategy based on a guided inquiry, on the adjustment of physics laboratory practices. The strategy was carried out in three schools of Bogotá (Colombia) in a research consisting of 145 students from secondary education, monitoring the interactions of students employing the follow-up process in the interaction whit laboratory practices model, and analyzing the progress of their performances inherent to the competences suggested in the research. From a mixed methodology, analytical descriptive, were implemented ad hoc designed input and output tests, which establish the level of appropriation of competencies, before and after the implementation of the methodological strategy. The results obtained show the effectiveness of the strategy within the educational contexts considered. It is concluded that the strategy presents an innovative and relevant character, since it proposes guiding and didactic elements for the teaching of science, bringing students closer to the new technological and scientific developments of today.
\end{abstract}

Keywords: guided inquiry, methodological strategy, scientific competences, laboratory practices, secondary education.

\section{Introducción}

A nivel mundial investigaciones educativas han establecido que, en las últimas décadas, el número de jóvenes que muestran interés por cursar carreras científicas ha contado con un notable descenso (Pedrinaci, Caamaño, Cañal, \& de Pro, 2012). La reducción en las tasas de matrículas en este tipo de carreras ha generado una creciente preocupación (Daza-Caicedo, et al., 2011), esto debido al impacto que la ciencia y la tecnología tienen en el desarrollo económico y social de los países.

Ante este hecho, se han adelantado estudios que permiten identificar los factores que influyen directamente en esta falta de motivación de los estudiantes. Entre ellos se destaca el estudio realizado por Sjøberg y Schereiner (2010), cuyos resultados muestran que la escasa intención de los adolescentes al elegir una profesión relacionada con ciencia, tecnología, ingeniería y matemáticas es producto de las dificultades presentadas en el aprendizaje de las ciencias y la baja incidencia de la educación científica en el pensamiento crítico.

Rocard, et al. (2008) identificaron que una de las razones por las que los jóvenes no desarrollan interés por las ciencias, es la presentación de programas sobrecargados y obsoletos, el acercamiento al conocimiento de manera abstracta, sin apoyo de la observación, ni la experimentación, dejando aún lado la relación directa con situaciones actuales y sus implicaciones sociales.

Por su parte, la Organización para la Cooperación y el Desarrollo Económico (OCDE) a través del Programa para la Evaluación Internacional de Alumnos conocida como prueba PISA (Programm for International Student Assessment), particularmente en la prueba del año 2006, cuya profundización se hizo en ciencias, además de centrarse en la medición del desempeño de los estudiantes y en la medición de los conocimientos 
a partir del concepto de alfabetización científica, incluyó preguntas para indagar acerca de las actitudes de los estudiantes hacia la ciencia y la tecnología desde tres áreas en particular: interés por la ciencia, apoyo a la investigación científica, sentido de la responsabilidad sobre los recursos y el ambiente. Todo ello considerando que para la OCDE estos aspectos presentan una relación con el rendimiento, la elección de carreras profesionales y el aprendizaje dado para toda la vida (OCDE, 2006). En este estudio se observó que, aunque los jóvenes tienen una alta valoración por los aportes efectuados por la ciencia, los cuales contribuyen significativamente al conocimiento del mundo, solo un $21 \%$ opina que cursaría una carrera científica (Gutiérrez, 2008). En la prueba de 2015, la gran mayoría de los estudiantes manifestaron un alto interés por temas científicos reconociendo su papel en el mundo, pero solo un $25 \%$ manifestó querer cursar una carrera relacionada con ciencias. Igualmente se identificó que el tiempo empleado para aprender ciencias y la forma como se enseñan, son cuestiones estrechamente relacionadas con el interés por cursar carreras científicas. Se observó también que los estudiantes con mejores resultados en esta área afirmaron que sus profesores de ciencias explican o exponen ideas científicas con mayor frecuencia, y adaptan esta enseñanza a sus necesidades (OCDE, 2016).

Desde el ámbito nacional, en Colombia se vienen aplicando varias encuestas de percepción sobre la ciencia y la tecnología. Específicamente en el año 2009, el Observatorio Colombiano de Ciencia y Tecnología (OCyT), en colaboración con la Secretaria Distrital de Educación (SED), realizó un estudio (Daza-Caicedo, et al., 2011) con 6.428 estudiantes de educación media en 28 colegios de Bogotá, 13 pertenecientes al sector oficial y 15 al sector privado. En este, se describen las percepciones que tienen los estudiantes sobre las profesiones científicas y las ingenierías, la opción laboral que estas ofrecen, la imagen que poseen de la ciencia y los científicos y, las diferentes valoraciones que los estudiantes hacen con relación al aporte de las materias de ciencias en sus vidas (Daza-Caicedo, et al., 2011). Con relación a los dos últimos aspectos, se destaca que uno de los factores que más valoración obtuvo, y que por ende se considera determinante en la falta de motivación hacia la elección de careras científicas, lo constituye la dificultad y el tedio que pueden acarrear las materias científicas.

Por otro lado, la falta de una adecuada alfabetización científica, que permita a los estudiantes cursar carreras en ciencias es evidenciado en los bajos desempeños en las evaluaciones internacionales (PISA) y nacionales (Saber 11). Concretamente, las pruebas PISA dan cuenta de que las competencias evaluadas en ciencias comprenden procesos cognitivos de especial significado y relevancia para su enseñanza (Bybee, 2004). Al respecto, específicamente en el año 2006, de los 57 países participantes solo el $38.59 \%$ alcanzó un puntaje por encima del promedio establecido por la OCDE (500), donde Colombia ocupó el puesto 53. En el año 2015, de los 70 países evaluados, solo el $42.85 \%$ obtuvo un resultado por encima del promedio de la OCDE (493), donde Colombia alcanzó el puesto 57 (OECD, 2007; OCDE, 2016). Estos porcentajes permiten establecer que el nivel de alfabetización cientifica a nivel mundial no llega ni al $50 \%$ de los paises participantes.

A nivel nacional, el Instituto Colombiano para la Evaluación de la Educación (ICFES) es el organismo encargado de evaluar, mediante exámenes estandarizados, la educación que reciben los colombianos en los diferentes niveles educativos. Específicamente la prueba SABER 11, evalúa la apropiación de competencias en estudiantes que culminan la educación media. Así, en cada una de las áreas se establecen cuatro niveles de desempeño siendo 4 el nivel más alto, donde el estudiante 
emplea conceptos, leyes o teorías en la solución de problemas a través de procedimientos, habilidades, conocimientos y un lenguaje propio de las ciencias. En el nivel de desempeño 1 (el más bajo), los estudiantes apenas alcanzan a reconocer información explicita y demuestran un insuficiente desarrollo de la competencia de indagación. Según los resultados obtenidos en ciencias en las pruebas Saber 11, aplicadas en los años 2015 y 2016, se observa que los estudiantes de colegios de calendario A, obtienen resultados que los ubican en el nivel de desempeño 2, mientras que los resultados de los estudiantes de colegios de calendario $\mathrm{B}$ los ubican en los niveles de desempeños 2 y 3 (ICFES, 2017).

En búsqueda de fortalecer la apropiación de competencias de carácter científico, la incorporación de procesos de indagación en la enseñanza de las ciencias promueve la implementación de prácticas en los estudiantes, donde se emplea el conocimiento teórico junto con destrezas y actitudes científicas potenciando de esta forma el desarrollo de este tipo de competencias (Crujeiras y Jiménez, 2015). Así, "el proceso educativo en las ciencias por indagación permite al estudiante valorar la curiosidad científica y la capacidad de análisis como fuente de aprendizaje, y utilizar el entorno cotidiano como un elemento cercano en la didáctica de las ciencias, idóneo para propiciar aprendizajes significativos" (Torres, 2010, p. 138). Todo esto, al interior de un escenario ideal como lo son los laboratorios (Högström, Ottander, y Benckert, 2010), donde las prácticas allí desarrolladas conducen a los estudiantes a espacios de construcción del conocimiento científico, de tipo conceptual, procedimental y actitudinal, relacionándolo con la cultura, la tecnología y la sociedad. Al respecto, Séré (2003) señala que los trabajos prácticos logran una conciencia epistemológica en los estudiantes, comprendiendo la variedad de relaciones entre teoría y experiencia en la experimentación, logrando un mayor impacto cuando la acción es interdisciplinaria.

Es así, como el propósito de este estudio se orientó hacia la implementación de una estrategia metodológica que no solo suscite una interacción más dinámica de los estudiantes en las clases de ciencias, que despierte la curiosidad y genere una apropiación verdadera del conocimiento científico, sino que promueva el desarrollo de competencias científicas específicamente en estudiantes de educación media, adoptando para ello una metodología de indagación aplicada a prácticas de laboratorio.

\section{Método}

De manera específica, este estudio se propone conocer en qué medida la indagación guiada como estrategia metodológica favorece el desarrollo de competencias en estudiantes de educación media.

\section{Diseño}

En coherencia se aplica la investigación-acción participativa (Álvarez-Gayou, 2003), de la mano del paradigma sociocrítico (Arnal, Del Rincón, y Latorre, 1992). El diseño se planteó desde una perspectiva mixta adoptando un enfoque descriptivo e interpretativo, e integrando la triangulación.

\section{Participantes}

La población queda representada por estudiantes y docentes de educación media de tres (3) instituciones de educación formal, ubicadas en la ciudad de Bogotá: Colegio Hunzá, Colegio San Simón y el Colegio Abraham Lincoln. Estas instituciones 
representan de forma general las características de los colegios de esta ciudad, teniendo en cuenta aspectos involucrados en el contexto educativo, presentes en cada una de las instituciones participantes. Estos centros educativos se encuentran ubicados en diferentes localidades y estratos socioeconómicos, poseen distintos calendarios académicos, algunos pertenecen al sector oficial o privado de la educación y presentan diferentes resultados a lo largo de su participación en pruebas SABER 11, tal y como se describe en la Tabla 1.

Tabla 1

Caracterización institucional población objeto de estudio.

\begin{tabular}{lccc}
\hline Parámetros / Colegio & Hunzá & San Simón & Abraham Lincoln \\
\hline Tipo de institución & Oficial & Privada & Privada \\
\hline Calendario académico & A & A & B \\
\hline Estratificación & $1-2$ & $3-4$ & $5-6$ \\
\hline $\begin{array}{l}\text { Categoría pruebas } \\
\text { SABER 11 (2016) }\end{array}$ & B & A+ & A+ \\
\hline $\begin{array}{l}\text { Puesto pruebas SABER } \\
11(2016)\end{array}$ & 764 & 278 & 26
\end{tabular}

La muestra (Tabla 2) está conformada por estudiantes de uno de los grupos de los grados décimo y undécimo de las tres instituciones educativas, considerando criterios de accesibilidad y disponibilidad de participación en el estudio.

Tabla 2

Muestra seleccionada

\begin{tabular}{lcccccc}
\hline $\begin{array}{l}\text { Colegio/ } \\
\text { Grados }\end{array}$ & Grado & $\begin{array}{c}\text { Hunzá } \\
\mathbf{N}^{\circ} \text { de } \\
\text { estudiantes }\end{array}$ & Grado & $\begin{array}{c}\text { San Simón } \\
\mathbf{N}^{\circ} \text { de } \\
\text { estudiantes }\end{array}$ & Grado & $\begin{array}{c}\text { Abraham Lincoln } \\
\text { estudiantes }\end{array}$ \\
\hline Décimo & 1001 & 36 & 1001 & 23 & $10 \mathrm{~A}$ & 16 \\
\hline Undécimo & 1101 & 35 & 1101 & 15 & $11 \mathrm{~A}$ & 20 \\
\hline Total & & 71 & & 38 & & 36 \\
\hline
\end{tabular}

\section{Instrumentos}

Para implementar la estrategia metodológica se utilizaron distintos instrumentos:

1. Una prueba de entrada que permitió indagar el nivel de apropiación de las competencias científicas que se fortalecerían con la implementación de la estrategia metodológica. El diseño de esta prueba a su vez se dividió en dos partes. La primera, incluyó preguntas con única respuesta, donde se indagó por la apropiación de tres las competencias propuestas en la investigación: uso de códigos particulares de la ciencia $(U C)$, procedimental y experimental (PE) y Pensamiento reflexivo y crítico de la ciencia $(P R C)$. Para ello se conformó un banco de 50 preguntas tomadas de pruebas estandarizadas aplicadas a nivel internacional (PISA) y nivel nacional (Saber 11) en los últimos 5 años que mostraban relación con las competencias propuestas y que permitieron orientar la forma como debían ser elaboradas. Los ítems fueron 
seleccionados a partir del análisis de material clave disponible en páginas virtuales ${ }^{1}$, proporcionado por entidades con relevancia en la materia. Después de un estudio riguroso se seleccionaron y adaptaron 15 preguntas a partir de las cuales se validó el nivel de apropiación de cada una de las competencias propuestas en la investigación (5 por cada competencia). Para la selección y redacción de las preguntas propuestas en la prueba, se tuvieron en cuenta principalmente, criterios de relevancia, claridad, coherencia, complejidad, pertinencia.

Para la validación de este instrumento se implementó una validez teórica dada a partir de la revisión de literatura expuesta en el marco teórico de la investigación y una validez de contenido efectuada a través del método de agregados individuales, donde jueces conformados por pares externos (3), revisaron las preguntas expuestas en la prueba. Los jueces ${ }^{2}$ seleccionados efectuaron la revisión de la prueba propuesta a partir de una rúbrica con las categorías que se tuvieron en cuenta en el diseño realizado. Para complementar el proceso de validación de la prueba, se consideró un grupo piloto, conformado por 10 estudiantes del colegio Abraham Lincoln (5 de grado 10B y 5 del grado 11B) y 10 estudiantes del colegio Hunzá (5 de 1102 y 5 de 1002). A partir de las observaciones efectuadas por los estudiantes se ajustó el tiempo asignado a la prueba y la edición de una de las gráficas propuestas.

La segunda parte de la prueba presentaba un cuestionario tipo Likert que indagaba acerca de las percepciones que los estudiantes poseían sobre el trabajo en grupo (cuarta competencia propuesta) asignando a los ítems propuestos valoraciones entre 1 y 5 , siendo 1 la valoración más baja y 5 la más alta de acuerdo con sus percepciones. Para la fiabilidad de este instrumento se aplicó un cuestionario inicial al grupo piloto y tras efectuar un proceso de depuración se obtuvo un alfa de Cronbach de .92 proponiendo un total 5 ítems para esta parte de la prueba.

2. Se utilizó también una ficha de programación de laboratorio, a partir de la que se organizaron las prácticas propuestas en cada institución educativa especificando temáticas, material, tipos de laboratorio a implementar y los espacios en los que se llevarían a cabo cada práctica de acuerdo con el horario de los distintos grupos.

3. Asimismo, se diseñó el instrumento rúbrica de progreso de las prácticas de laboratorio, para registrar los avances o dificultades detectadas en los estudiantes al interactuar con la metodología propuesta. A través de esta rúbrica se adjudicaban valoraciones a desempeños involucrados en cada competencia.

4. Por último, se utilizó una prueba de salida, implementada para establecer el impacto de la estrategia metodológica a partir de los avances en las competencias científicas que se buscó desarrollar en la investigación. El procedimiento adoptado para

\footnotetext{
${ }^{1}$ Direcciones electrónicas pruebas PISA: http://educalab.es/inee/evaluaciones-internacionales/preguntasliberadas-pisa-piaac/preguntas-pisa-ciencias/fisica;http://educalab.es/inee/evaluacionesinternacionales/preguntas-liberadas-pisa-piaac/pisa-por-ordenador; http://www.mecd.gob.es/dctm/ievaluacion/internacional/ciencias-en-pisa-paraweb.pdf?documentId=0901e72b8072f577; www.oei.es/evaluacioneducativa/tipo.pdf Direcciones electrónicas pruebas Saber 11: http://Cuadernillo\%20de\%20 preguntas-Saber\%2011\%20Ciencias\%20naturales.pdf; http://Guia\%20de\%20orientacion\%20saber\%2011-2017-1\%20(2).pdf; https://orientacion.universia.net.co/imgs2011/imagenes/cuadernill-2016_11_03_145738.pdf

2 Jurados: Docente líder del Centro de Investigación y docente de Teoría del Conocimiento del colegio Abraham Lincoln; Docente de Física del colegio Abraham Lincoln; Docente Universitaria y Doctora en Educación.
} 
la elaboración y validación de este instrumento fue el mismo que se adoptó para la prueba de entrada.

\section{Procedimiento}

En la fase de diseño de la implementación se partió de un diagnóstico previo, que permitió establecer la apropiación del enfoque por competencias en las instituciones objeto de estudio, realizado a través de entrevistas a rectores y docentes, el estudio de documentos institucionales (Proyecto Educativo Institucional (PEI), documentos del área de ciencias naturales y planes de estudio), y la aplicación de una encuesta sociodemográfica que permitió identificar aspectos socioeconómicos, familiares e interés de los estudiantes por la ciencia.

Posteriormente, según la revisión de la literatura, se optó por establecer las competencias que se fortalecerían en la investigación, cada una con sus respectivos desempeños: Uso de códigos particulares de la ciencia (UC), Procedimental Experimental (PE), Pensamiento reflexivo y crítico de la ciencia (PRC), y Trabajo en equipo (TE) ${ }^{3}$.

En esta fase de diseño se propuso un modelo de prácticas de laboratorio bajo la metodología de indagación (Benarroch, 2015, Caamaño, 2012), que considera la implementación de prácticas experimentales a través de una serie de fases: 1) Fase de focalización; 2) Fase de exploración; 3) Fase de comprobación y contraste; 4) Fase de socialización de resultados; y, 5) Fase de aplicación. El proceso abarca la exploración de ideas previas sobre alguna temática, la formulación de hipótesis, el planteamiento de procedimientos, la confrontación de predicciones sobre los resultados obtenidos abordados de forma lógica y argumentativa. Posteriormente el conocimiento es expuesto y difundido para poder aplicarse en nuevas situaciones en las que sea transferible.

Concretamente y para llevar a cabo este diseño, en una primera fase se aplicó la prueba de entrada en cada uno de los grupos de las tres instituciones educativas. Una vez recolectada esta información, se procedió a la tabulación y codificación de los resultados para efectuar su respectivo análisis.

En una segunda fase, en reunión con los docentes titulares, se socializaron y analizaron los resultados de la prueba de entrada obtenidos en cada grado. Asimismo, se efectúo el plan de trabajo que se llevaría a cabo en cada grado a través del instrumento ficha de programación de laboratorio, estableciendo las prácticas de

3 Uso de códigos particulares de la ciencia $(U C)$ que hace referencia a la socialización del conocimiento, la utilización de códigos particulares de comunicación a través de los cuales se propone la construcción de estructuras semánticas que pueden ser interpretadas por los miembros de una comunidad; Procedimental - Experimental (PE) que resalta la contrastación de lo teórico con la realidad a través de la experimentación mediante la implementación de una serie de procedimientos que implican destrezas relacionadas con manipulación de material, elaboración de montajes, uso apropiado de instrumentos y tratamiento de datos, manipulación de herramientas computarizadas y material tecnológico y la realización de algunas actividades que implican el seguimiento de instrucciones por parte de los estudiantes, entre otras; Pensamiento reflexivo y crítico de la ciencia $(P R C)$, resalta que la solución de situaciones problemáticas, implican la integración de saberes en forma propositiva y creativa, para lo cual se requiere de un adecuado desarrollo de los procesos cognitivos que implican procesos básicos de pensamiento permitiendo a los estudiantes no solo analizar y explicar fenómenos científicos, sino construir conocimiento a partir de ellos; y Trabajo en equipo (TE), desarrolla la habilidad para interactuar con los demás en forma grupal e individual, despertando sentido de pertenencia, responsabilidad y eficiencia al efectuar confrontaciones de ideas, celebrar acuerdos y abordar tareas de manera conjunta. 
laboratorio que se desarrollaron de acuerdo con el plan de estudios de cada asignatura. Por último, se programaron sesiones de encuentro con los docentes titulares para retroalimentar el proceso y aspectos del desarrollo de las prácticas.

La tercera ${ }^{4}$ fase dio paso a la realización de prácticas de laboratorio bajo la nueva metodología. Una vez terminada cada práctica se efectuó la revisión de los planteamientos expuestos por los estudiantes a partir del instrumento rúbrica de progreso prácticas de laboratorio con el cual se asignaba una valoración específica a los desempeños propuestos en las diferentes prácticas experimentales para cada una de las competencias. Una vez terminada la interacción de los estudiantes con la estrategia implementada se efectuó un análisis de los progresos alcanzados en cada competencia desde las valoraciones obtenidas en los desempeños propuestos en cada una de las prácticas experimentales.

En una cuarta fase se llevó a cabo la aplicación del instrumento prueba final con el cual, en una primera parte, se estableció nuevamente el nivel de apropiación de competencias científicas alcanzado, posterior a la implementación de la estrategia metodológica. En la segunda parte se implementó el cuestionario Likert utilizado en la prueba de entrada para identificar algún cambio en las apreciaciones de los estudiantes a cerca del trabajo en equipo.

Finalmente, en una quinta fase se analizaron los resultados tanto de la prueba de entrada, como de la prueba de salida a través de un análisis de varianza que permitiera establecer el impacto de la propuesta.

\section{Resultados}

Sobre los resultados obtenidos en la prueba de entrada, se obtuvo inicialmente el promedio de las respuestas dadas, a partir de las situaciones propuestas desde cada competencia, obteniéndose un promedio general (Tablas 3 y 4).

Tabla 3

Desempeños de los estudiantes de grado décimo en las competencias evaluadas en la prueba de entrada

\begin{tabular}{cllcccc}
\hline Colegio & \multicolumn{2}{c}{ Hunzá } & \multicolumn{2}{c}{ San Simón } & \multicolumn{2}{c}{ Abraham Lincoln } \\
\hline Competencia & Correctas & Incorrectas & Correctas & Incorrectas & Correctas & Incorrectas \\
\hline UC & $36.56 \%$ & $63.44 \%$ & $53.92 \%$ & $46.08 \%$ & $66.26 \%$ & $33.74 \%$ \\
\hline PE & $33.14 \%$ & $66.86 \%$ & $47.84 \%$ & $52.16 \%$ & $73.78 \%$ & $26.22 \%$ \\
\hline PRC & $33.14 \%$ & $66.86 \%$ & $48.68 \%$ & $51.32 \%$ & $76.28 \%$ & $23.72 \%$ \\
\hline Promedio & $34.28 \%$ & $65.72 \%$ & $50.14 \%$ & $49.85 \%$ & $72.10 \%$ & $27.89 \%$ \\
\hline
\end{tabular}

Nota: UC: Competencia Uso de códigos particulares de la Ciencia; PE: Competencia Procedimental-Experimental; PRC: Competencia Pensamiento reflexivo y crítico de la ciencia.

\footnotetext{
${ }^{4}$ Estas prácticas fueron desarrolladas por una de las investigadoras, quien estuvo acompañada, durante cada sesión de clase, por el docente titular de la asignatura, el cual presentó una actitud de interés y entusiasmo, pues la forma como se llevaron a cabo las actividades experimentales connotaron asombro e interés por el conocimiento; del mismo modo, poco a poco se iban recogiendo impresiones de cada sesión, que fueron socializadas en las reuniones de encuentro programadas a fin de obtener la retroalimentación necesaria para nutrir aspectos de la implementación.
} 
Tabla 4

Desempeños de los estudiantes de grado undécimo en las competencias evaluadas en la prueba de entrada

\begin{tabular}{ccccccc}
\hline Colegio & \multicolumn{2}{c}{ Hunzá } & \multicolumn{2}{c}{ San Simón } & \multicolumn{2}{c}{ Abraham Lincoln } \\
\hline Competencia & Correctas & Incorrectas & Correctas & Incorrectas & Correctas & Incorrectas \\
\hline UC & $47.68 \%$ & $52.32 \%$ & $55.98 \%$ & $44.02 \%$ & $76.00 \%$ & $24.00 \%$ \\
\hline PE & $34.62 \%$ & $65.38 \%$ & $51.98 \%$ & $48.02 \%$ & $70.00 \%$ & $30.00 \%$ \\
\hline PRC & $36.14 \%$ & $63.86 \%$ & $49.63 \%$ & $50.64 \%$ & $68.00 \%$ & $32.00 \%$ \\
\hline Promedio & $39.48 \%$ & $60.52 \%$ & $52.53 \%$ & $47.47 \%$ & $71.33 \%$ & $28.66 \%$ \\
\hline
\end{tabular}

Nota: UC: Competencia Uso de códigos particulares de la Ciencia; PE: Competencia Procedimental-Experimental; PRC:

Competencia Pensamiento reflexivo y crítico de la ciencia

A partir de los resultados obtenidos en la prueba de entrada (Tabla 3), puede observarse que los estudiantes del colegio Abraham Lincoln presentaron un mayor nivel de apropiación de las competencias a fortalecer en la investigación tanto en el grupo de décimo como en el de undécimo. Lo anterior, puede atribuirse, en cierta medida, al tipo de formación ofrecida por esta institución (Bachillerato IB) donde constantemente se privilegia la metodología de indagación no solamente al interior de prácticas experimentales, aspecto evidenciado en el diagnóstico al comienzo de la investigación. Así mismo se observó que los bajos resultados obtenidos por los estudiantes tanto de grado décimo como de undécimo del colegio Hunzá, en cada competencia, fueron similares, al igual que las valoraciones a nivel general. Algo similar se presentó en los estudiantes de grado décimo y undécimo del colegio San Simón, sin embargo, el promedio general de los resultados en esta última institución fue más aceptables, sin dejar de evidenciarse que no hay una adecuada apropiación de las competencias propuestas para la investigación ${ }^{5}$.

Con respecto a la segunda parte de la prueba que indagaba las percepciones de los estudiantes con relación al trabajo grupal (competencia $T E$ ), las valoraciones de 1 a 5 fueron agrupadas en: valoración baja (1 a 2), valoración aceptable (3) y valoración alta (4 a 5) (Tabla 5).

Tabla 5

Percepción del trabajo en equipo (competencia TE) prueba de entrada

\begin{tabular}{lccccccc}
\hline Colegio & \multicolumn{1}{c}{ Grado } & \multicolumn{3}{c}{ Décimo } & \multicolumn{3}{c}{ Undécimo } \\
\cline { 2 - 7 } & $\begin{array}{c}\text { Valoración } \\
\text { Ítem }\end{array}$ & Baja & Aceptable & Alta & Baja & Aceptable & Alta \\
& 1 & $11.4 \%$ & $20.0 \%$ & $68.6 \%$ & $7.7 \%$ & $26.9 \%$ & $65.4 \%$ \\
\hline \multirow{3}{*}{ Hunzá } & 2 & $5.7 \%$ & $20.0 \%$ & $74.3 \%$ & $3.8 \%$ & $26.9 \%$ & $69.2 \%$ \\
\cline { 2 - 7 } & 3 & $8.5 \%$ & $20.0 \%$ & $71.5 \%$ & $11.5 \%$ & $7.7 \%$ & $80.8 \%$ \\
\cline { 2 - 7 } & 4 & $11.4 \%$ & $22.9 \%$ & $65.7 \%$ & $15.3 \%$ & $19.2 \%$ & $65.4 \%$ \\
\hline San & 5 & $2.9 \%$ & $17.1 \%$ & $80.0 \%$ & $3.8 \%$ & $26.9 \%$ & $69.2 \%$ \\
\hline
\end{tabular}

${ }^{5}$ Lo expuesto en consonancia con lo evidenciado en el diagnóstico previo, llevado a cabo en estas instituciones educativas, el cual mostró la baja incidencia de actividades tendientes a desarrollar competencias científicas y la ausencia de la apropiación de metodologías de indagación. 


\begin{tabular}{lccccccc}
\hline Simón & 2 & $8.7 \%$ & $26.1 \%$ & $65.2 \%$ & $13.3 \%$ & $20.0 \%$ & $66.7 \%$ \\
\cline { 2 - 7 } & 3 & $26.0 \%$ & $4.3 \%$ & $69.5 \%$ & $6.7 \%$ & $13.3 \%$ & $80.0 \%$ \\
\cline { 2 - 7 } & 4 & $17.3 \%$ & $34.8 \%$ & $47.8 \%$ & $0.0 \%$ & $20.0 \%$ & $80.0 \%$ \\
\hline \multirow{3}{*}{$\begin{array}{l}\text { Abraham } \\
\text { Lincoln }\end{array}$} & $\mathbf{5}$ & $8.7 \%$ & $21.7 \%$ & $69.6 \%$ & $6.7 \%$ & $6.7 \%$ & $86.6 \%$ \\
\cline { 2 - 7 } & 2 & $0.0 \%$ & $6.3 \%$ & $93.8 \%$ & $15.0 \%$ & $10.0 \%$ & $75.0 \%$ \\
\cline { 2 - 7 } & 3 & $6.0 \%$ & $6.3 \%$ & $93.8 \%$ & $5.0 \%$ & $5.0 \%$ & $90.0 \%$ \\
\hline
\end{tabular}

A partir de los resultados obtenidos, se observó que en los grados décimo de las instituciones Hunzá y San Simón, y en los grados undécimos de las instituciones Hunzá y Abraham Lincoln se debía efectuar un trabajo más riguroso en relación con las dinámicas del trabajo grupal. No obstante, aunque el porcentaje de estudiantes que asignaban valoraciones bajas y aceptables a los ítems indagados en su gran mayoría no superaban el 35\%, al implementar la propuesta se persiguió que este porcentaje disminuyera.

Con respecto a los progresos de los estudiantes en cada competencia para las prácticas analizadas, y de acuerdo con la Figura 1, se observó que, en el colegio Hunzá, específicamente para el grado 1001, aunque las valoraciones iniciales no fueron las mismas en cada competencia, se presentó mayor avance en la competencia $U C$. La competencia que menos avance tuvo fue $P R C$, lo que concuerda con debilidades observadas al interior de las guías, relacionadas estas con el planteamiento de predicciones, la presentación de argumentos que daba cuenta de la validez del conjunto de datos recogidos, la elaboración adecuada de conclusiones y la identificación del objetivo de la práctica.

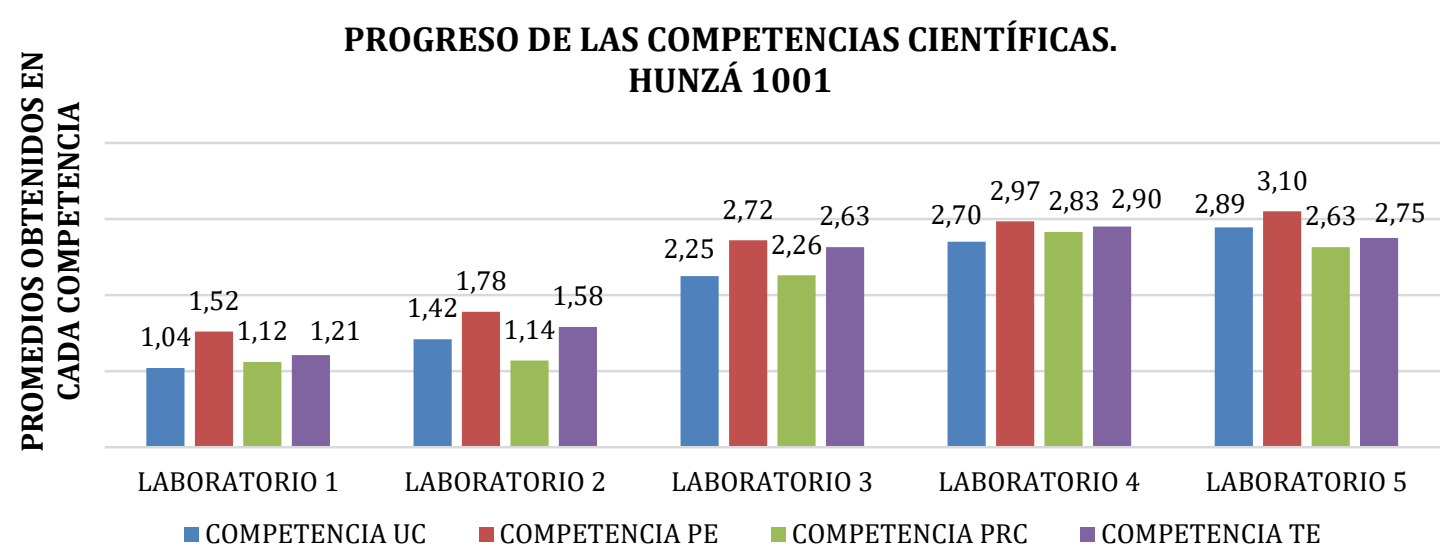

Figura 1. Progreso de las competencias Colegio Hunzá 1001

Nota: UC: Competencia Uso de códigos particulares de la Ciencia; PE: Competencia Procedimental-Experimental; PRC: Competencia Pensamiento reflexivo y crítico de la ciencia.

En esta misma institución para el grado 1101, de acuerdo con la Figura 2, se observó que los promedios de las competencias fueron muy similares en cada práctica de laboratorio. Al igual que en el grado 1001, se presentó mayor avance en la competencia $U C$. La competencia que menos progreso reveló fue $P E$, aspecto que estuvo acorde con debilidades observadas al interior de las guías presentadas, 
relacionadas estas con la elaboración adecuada de predicciones, el planteamiento de procedimientos que permitan la recolección de información para validar una predicción y la organización adecuada de información a través de tablas.

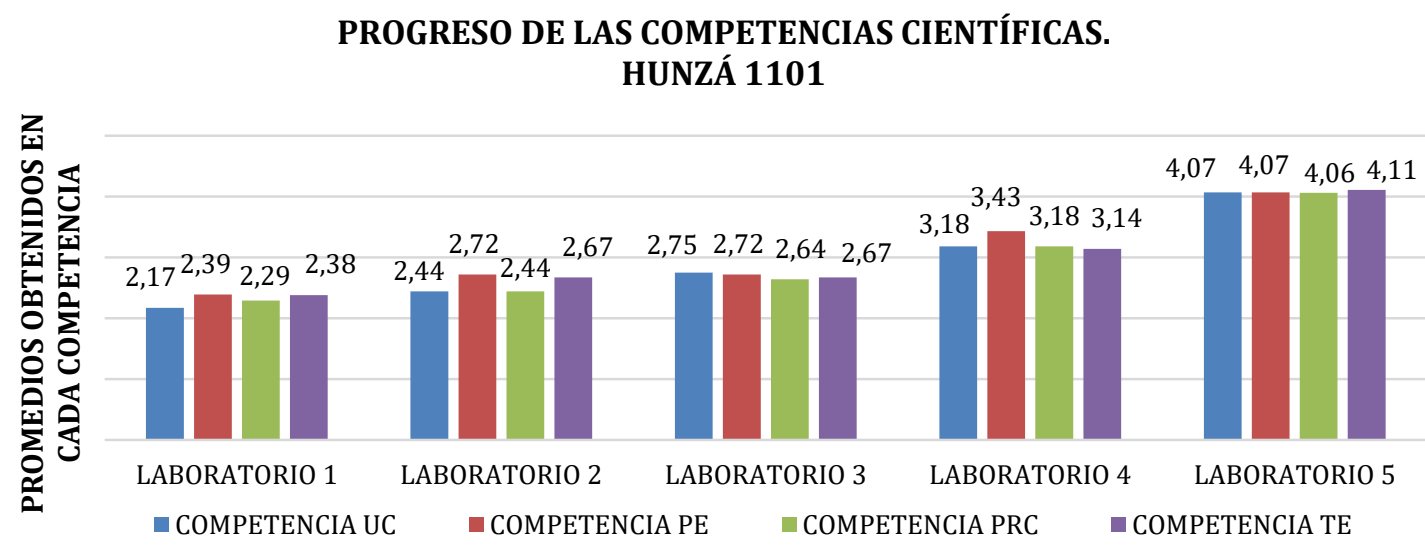

Figura 2. Progreso de las competencias Colegio Hunzá 1101

Nota: UC: Competencia Uso de códigos particulares de la Ciencia; PE: Competencia Procedimental-Experimental; PRC: Competencia Pensamiento reflexivo y crítico de la ciencia.

En el colegio San Simón, de acuerdo con los resultados expuestos en las Figuras 3 y 4, se observó que, aunque las valoraciones en cada competencia no fueron similares entre sí, su progreso fue mayor que el evidenciado en cada uno de los grados del colegio Hunzá. Específicamente en el grado 1001, la competencia que mayor avance obtuvo fue $P R C$. La competencia que menor avance reflejó fue $U C$ asociada con dificultades relacionadas específicamente con el uso de un adecuado lenguaje científico en la explicación de fenómenos y la contextualización de la práctica.

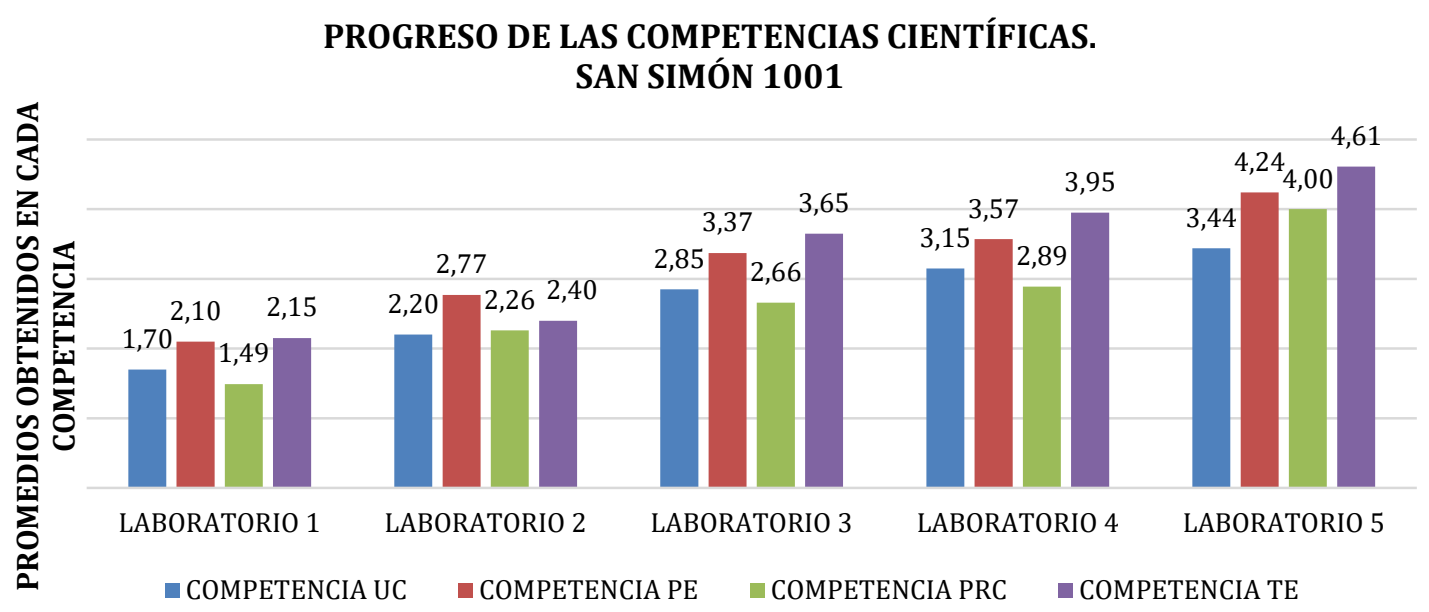

Figura 3. Progreso de las competencias Colegio San Simón 1001

Nota: UC: Competencia Uso de códigos particulares de la Ciencia; PE: Competencia Procedimental-Experimental; PRC: Competencia Pensamiento reflexivo y crítico de la ciencia. 
En el grado 1101 del colegio San Simón, se evidenció que al igual que en el grado 1001, la competencia con mayor progreso fue $P R C$. La competencia con menor progreso fue $P E$ asociada a dificultades observadas al interior de las guías relacionadas con la elaboración de predicciones y el planteamiento de procedimientos que permitan recolectar información para validar una hipótesis.

\section{PROGRESO DE LAS COMPETENCIAS CIENTÍFICAS. SAN SIMÓN 1101}

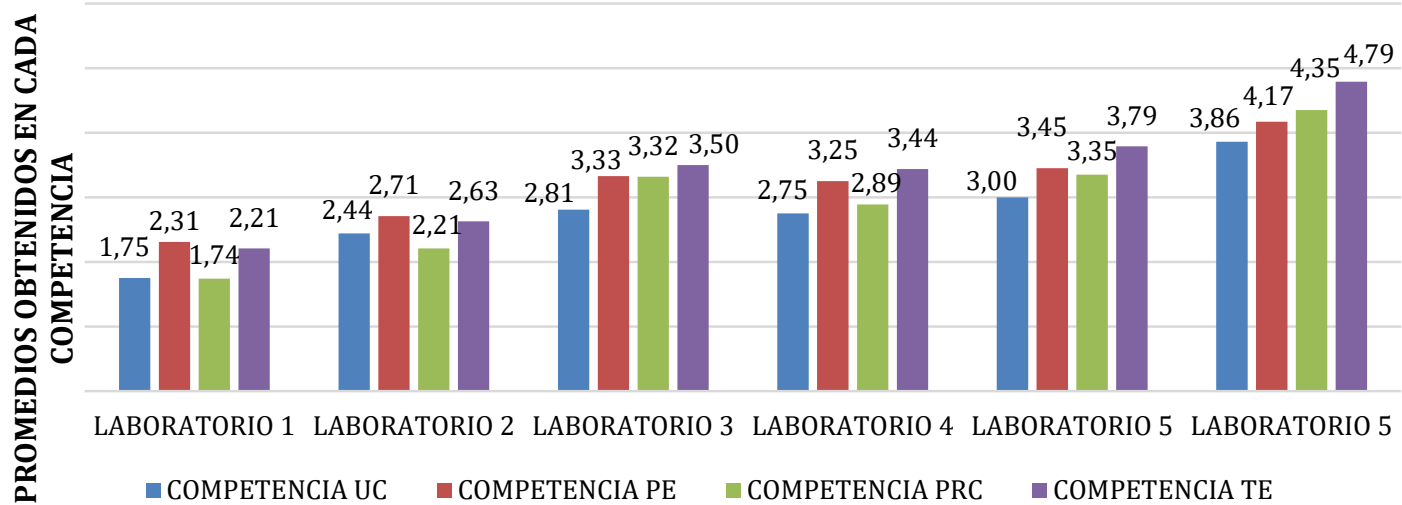

Figura 4. Progreso de las competencias Colegio San Simón 1101

Nota: UC: Competencia Uso de códigos particulares de la Ciencia; PE: Competencia Procedimental-Experimental; PRC:

Competencia Pensamiento reflexivo y crítico de la ciencia.

De otra parte, el colegio Abraham Lincoln fue la institución educativa donde se dio un menor progreso en las competencias, aunque debe resaltarse que las valoraciones asignadas en cada una de ellas fueron las más altas.

Como lo muestra la Figura 5, específicamente en el grado 10A se dio un mayor avance en la competencia $T E$ mientras que, la competencia con menor progreso fue $U C$ asociado con dificultades observadas al interior de las guías específicamente con la elaboración de algunos argumentos.

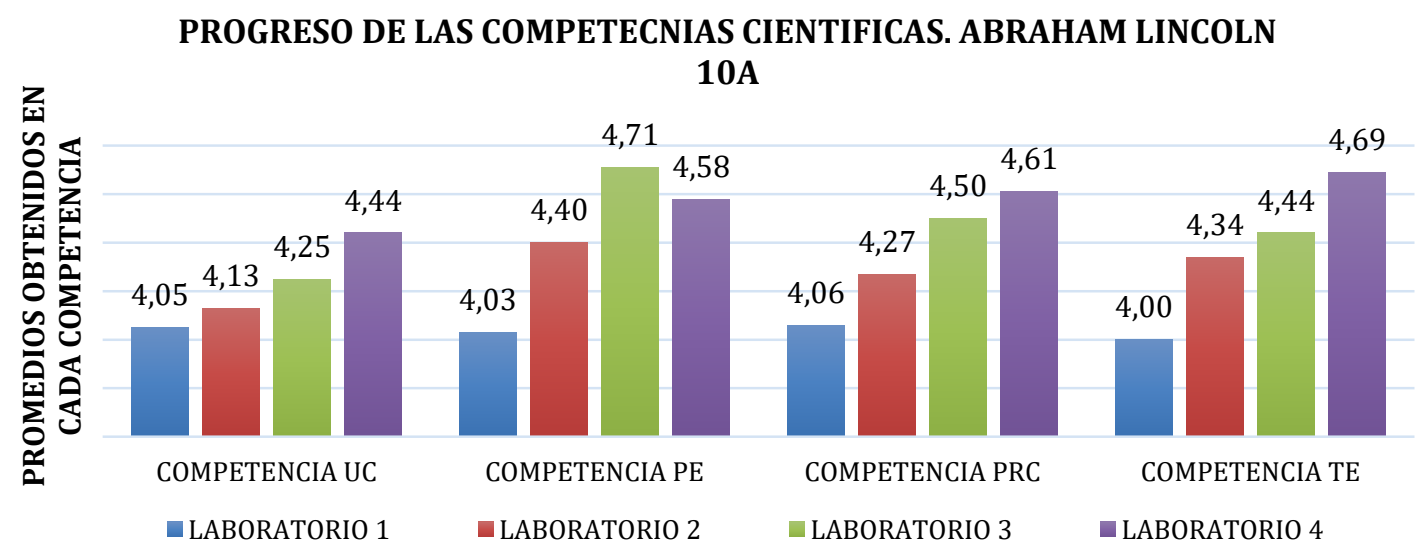

Figura 5. Progreso de las competencias Colegio Abraham Lincoln 10A.

Nota: UC: Competencia Uso de códigos particulares de la Ciencia; PE: Competencia Procedimental-Experimental; PRC: Competencia Pensamiento reflexivo y crítico de la ciencia. 
Puntualmente en el grado 11A, se encuentra un mayor avance en la competencia $P R C$ y en forma similar, la que menor progreso obtuvo fue $U C$ asociada también a dificultades observadas al interior de las guías, en relación con lo que compete a la elaboración de argumentos (Figura 6).

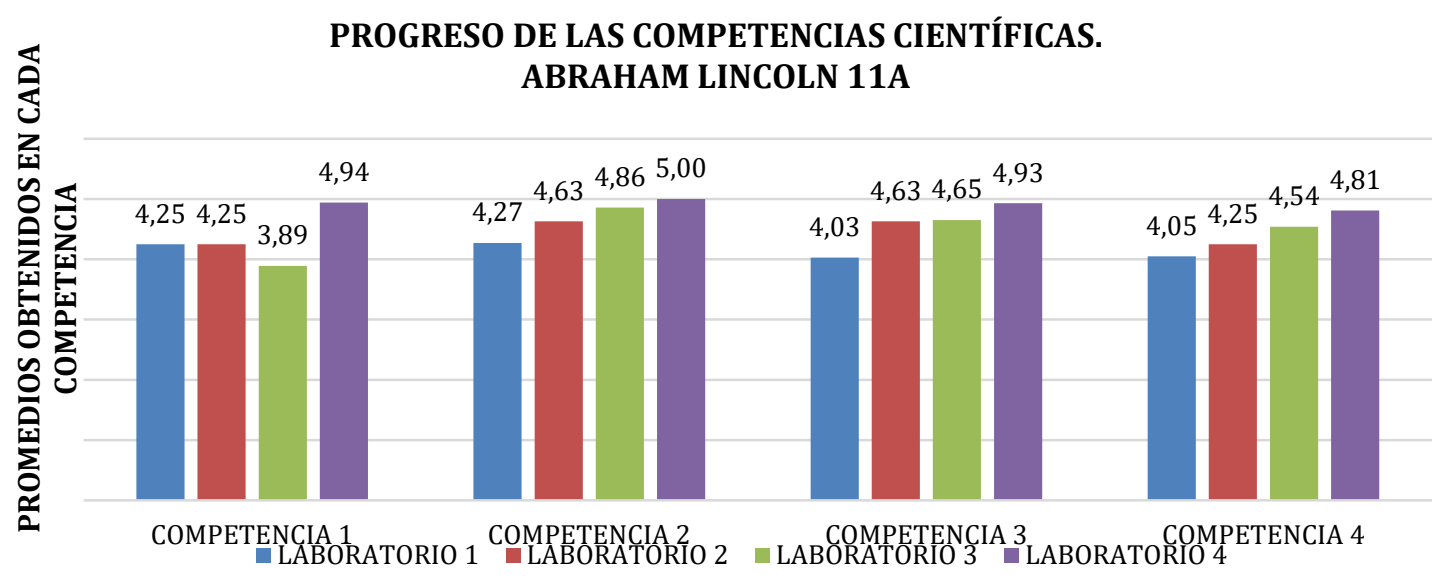

Figura 6. Progreso de las competencias Colegio Abraham Lincoln 11A

Nota: UC: Competencia Uso de códigos particulares de la Ciencia; PE: Competencia Procedimental-Experimental; PRC:

Competencia Pensamiento reflexivo y crítico de la ciencia.

Por último, la retroalimentación de las debilidades y fortalezas en las producciones de los estudiantes antes de iniciar una nueva práctica posibilitó que se dieran avances más significativos en los desempeños al interior de las nuevas prácticas.

Con relación a la tabulación de los resultados obtenidos en la prueba de salida, en forma similar a la prueba de entrada, se obtuvo el promedio de las respuestas dadas a partir de las situaciones propuestas en cada competencia (Tablas 6 y 7).

Tabla 6

Desempeños de los estudiantes de grado décimo en las competencias evaluadas en la prueba de salida

\begin{tabular}{ccccccc}
\hline Colegio & \multicolumn{2}{c}{ Hunzá } & \multicolumn{2}{c}{ San Simón } & \multicolumn{2}{c}{ Abraham Lincoln } \\
\hline Competencia & Correctas & Incorrectas & Correctas & Incorrectas & Correctas & Incorrectas \\
\hline UC & $65.15 \%$ & $34.85 \%$ & $69.57 \%$ & $30.43 \%$ & $72.5 \%$ & $27.4 \%$ \\
\hline PE & $56.0 \%$ & $44.0 \%$ & $59.13 \%$ & $40.87 \%$ & $82.5 \%$ & $17.5 \%$ \\
\hline PRC & $37.14 \%$ & $62.86 \%$ & $55.65 \%$ & $44.35 \%$ & $76.25 \%$ & $23.75 \%$ \\
\hline Promedio & $52.76 \%$ & $47.24 \%$ & $61.45 \%$ & $38.55 \%$ & $77.08 \%$ & $22.92 \%$ \\
\hline
\end{tabular}

Tabla 7

Desempeños de los estudiantes de grado undécimo en las competencias evaluadas en la prueba de salida

\begin{tabular}{ccccccc}
\hline Colegio & \multicolumn{2}{c}{ Hunzá } & \multicolumn{2}{c}{ San Simón } & \multicolumn{2}{c}{ Abraham Lincoln } \\
\hline Competencia & Correctas & Incorrectas & Correctas & Incorrectas & Correctas & Incorrectas \\
\hline UC & $71.54 \%$ & $28.46 \%$ & $76.0 \%$ & $24.0 \%$ & $87.0 \%$ & $13.0 \%$ \\
\hline PE & $53.08 \%$ & $46.92 \%$ & $68.0 \%$ & $32.0 \%$ & $82.0 \%$ & $18.0 \%$ \\
\hline
\end{tabular}




\begin{tabular}{ccccccc}
\hline PRC & $40.0 \%$ & $60.0 \%$ & $54.67 \%$ & $45.33 \%$ & $73.0 \%$ & $27.0 \%$ \\
\hline Promedio & $54.87 \%$ & $45.13 \%$ & $66.22 \%$ & $33.78 \%$ & $80.7 \%$ & $19.3 \%$ \\
\hline
\end{tabular}

Examinando los resultados obtenidos en la prueba de salida, nuevamente los estudiantes del colegio Abraham Lincoln presentan las valoraciones más altas a pesar de que, como se mencionó, su progreso en las competencias durante la interacción con la estrategia metodológica no fue tan significativo como con los otros dos colegios.

Igualmente se observó que los resultados obtenidos por los estudiantes del colegio Hunzá, en ambos grados, presentan un aumento significativo. Destacándose el desempeño referente a la competencia $U C$ cuya valoración es más alta, lo cual concuerda con el progreso evidenciado durante la interacción con la estrategia metodológica.

De otro lado, aunque los estudiantes del colegió San Simón también mostraron un aumento significativo en los resultados, debe anotarse que, en forma particular, aquellas competencias que mostraron un progreso significativo durante la interacción con la nueva estrategia metodológica, fueron las que obtuvieron resultados más bajos en la prueba de salida, lo cual podría adjudicarse al elevado nivel de complejidad de las preguntas propuestas en esta competencia, pues como se observa, en todos los grados de las instituciones educativas, la competencia $P R C$ fue en donde se presentaron las valoraciones más bajas, a excepción del grado 10A del colegio Abraham Lincoln. Lo que indica que definitivamente a los estudiantes se les dificulta efectuar procesos mentales avanzados en donde se requiere de un mayor nivel de análisis y deducción. Por último, se destaca que la competencia que mayor valoración presentó fue $U C$ en todos los grados de las tres instituciones educativas.

A partir de los resultados obtenidos en la primera parte de la prueba de entrada y la prueba de salida, se efectuó el análisis estadístico $\mathrm{T}$ Student ${ }^{6}$ que permitió realizar una prueba de hipótesis y con ella establecer el impacto de la metodología propuesta.

De esta forma las hipótesis que se buscaron comprobar fueron:

$\mathrm{H}_{\mathrm{O}}$ :

- El promedio de los resultados obtenidos en la prueba de entrada es mayor o igual que el promedio de los resultados obtenidos en la prueba de salida

$\mathrm{H}_{\mathrm{a}}$ :

- $\quad$ El promedio de los resultados obtenidos en la prueba de salida es mayor que el promedio de los resultados obtenidos en la prueba de entrada

Para llevar a cabo este análisis estadístico se empleó el programa computacional SPSS, con el que inicialmente se efectuó una prueba de normalidad a través de la prueba no paramétrica de Kolmogorov-Smirnov.

Por último, para establecer si se adoptaba la Hipótesis nula $\left(\mathrm{H}_{\mathrm{O}}\right)$ o la Hipótesis alterna $\left(\mathrm{H}_{\mathrm{a}}\right)$ se determinó, con el mismo programa computacional SPSS, el respectivo Pvalor para cada uno de los grados, descrito en la Tabla 8. Para ello se definió un nivel de significancia del $5 \%$ es decir $\alpha=.05$, estableciéndose de esta forma un nivel de confianza del $95 \%$.

\footnotetext{
${ }^{6}$ Se empleó este análisis estadístico al contar con variables cuantitativas (resultados de las pruebas de entrada y pruebas de salida) del mismo grupo (en cada grado), al que se le practicaron mediciones antes y después.
} 
Tabla 8

Cálculo del P-valor

\begin{tabular}{lcc}
\hline & Décimo & Undécimo \\
\cline { 2 - 3 } & P-valor & P-valor \\
\hline Hunzá & .0006 & .0007 \\
\hline San Simón & .0040 & .0080 \\
\hline Abraham Lincoln & .261 & .086 \\
\hline
\end{tabular}

Nota: Para $\mathrm{P}<\alpha=0.05$ se rechaza la hipótesis nula y se acepta la hipótesis alterna

Es así como se observó que el P-valor conseguido para los grados de los colegios Hunzá y San Simón indican que la implementación de la metodología propuesta fue viable para el fortalecimiento de las competencias científicas. No siendo así en los grados del colegio Abraham Lincoln, lo cual pudo estar relacionado con el hecho de que este grupo al poseer una formación en metodologías de indagación no mostró un cambio significativo en los resultados de las pruebas finales.

Ahora bien, retomando la segunda parte de la prueba de salida, que da cuenta de las apreciaciones de los estudiantes frente al trabajo grupal, aplicando nuevamente el cuestionario propuesto en la prueba de entrada y, agrupando las valoraciones dadas por los estudiantes de 1 a 5 en: valoración baja ( 1 a 2), valoración aceptable (3) y valoración alta (4 a 5), los datos obtenidos se registraron en la Tabla 9.

Tabla 9

Percepción del trabajo en equipo (competencia TE) prueba de salida

\begin{tabular}{|c|c|c|c|c|c|c|c|}
\hline \multirow[t]{2}{*}{ Colegio } & \multirow{2}{*}{$\begin{array}{c}\text { Grado } \\
\text { Valoración } \\
\text { Ítem }\end{array}$} & \multicolumn{3}{|c|}{ Décimo } & \multicolumn{3}{|c|}{ Undécimo } \\
\hline & & Baja & Aceptable & Alta & Baja & Aceptable & Alta \\
\hline \multirow{5}{*}{ Hunzá } & 1 & $0.0 \%$ & $11.4 \%$ & $88.6 \%$ & $0.0 \%$ & $3.8 \%$ & $96.1 \%$ \\
\hline & 2 & $2.9 \%$ & $14.9 \%$ & $82.9 \%$ & $0.0 \%$ & $19.2 \%$ & $80.7 \%$ \\
\hline & 3 & $5.7 \%$ & $14.3 \%$ & $80.0 \%$ & $0.0 \%$ & $3.8 \%$ & $96.1 \%$ \\
\hline & 4 & $5.7 \%$ & $14.3 \%$ & $80.0 \%$ & $0.0 \%$ & $19.2 \%$ & $80.7 \%$ \\
\hline & 5 & $5.7 \%$ & $5.7 \%$ & $88.6 \%$ & $0.0 \%$ & $7.7 \%$ & $92.3 \%$ \\
\hline \multirow{5}{*}{$\begin{array}{l}\text { San } \\
\text { Simón }\end{array}$} & 1 & $0.0 \%$ & $8.7 \%$ & $91.3 \%$ & $0.0 \%$ & $13.3 \%$ & $86.6 \%$ \\
\hline & 2 & $4.3 \%$ & $17.4 \%$ & $78.3 \%$ & $0.0 \%$ & $0.0 \%$ & $100.0 \%$ \\
\hline & 3 & $0.0 \%$ & $13.0 \%$ & $86.9 \%$ & $6.7 \%$ & $0.0 \%$ & $93.3 \%$ \\
\hline & 4 & $8.7 \%$ & $21.7 \%$ & $69.6 \%$ & $0.0 \%$ & $26.7 \%$ & $73.3 \%$ \\
\hline & 5 & $0.0 \%$ & $21.7 \%$ & $78.3 \%$ & $0.0 \%$ & $0.0 \%$ & $100.0 \%$ \\
\hline \multirow{5}{*}{$\begin{array}{l}\text { Abraham } \\
\text { Lincoln }\end{array}$} & 1 & $0.0 \%$ & $6.3 \%$ & $93.8 \%$ & $0.0 \%$ & $15.0 \%$ & $85.0 \%$ \\
\hline & 2 & $0.0 \%$ & $0.0 \%$ & $100.0 \%$ & $10.0 \%$ & $5.0 \%$ & $85,0 \%$ \\
\hline & 3 & $0.0 \%$ & $12.5 \%$ & $87.6 \%$ & $5.0 \%$ & $15.0 \%$ & $80.0 \%$ \\
\hline & 4 & $0.0 \%$ & $6.3 \%$ & $93.8 \%$ & $10.0 \%$ & $15.0 \%$ & $75.0 \%$ \\
\hline & 5 & $0.0 \%$ & $6.3 \%$ & $93.8 \%$ & $15.0 \%$ & $0.0 \%$ & $75.0 \%$ \\
\hline
\end{tabular}

Al efectuar el análisis de los resultados obtenidos en la prueba de salida se encontró que el porcentaje de estudiantes que asignó valoraciones bajas y aceptables a cada uno de los ítems disminuyó significativamente, lo que evidencia una valoración más alta frente a las dinámicas dadas al interior de un trabajo grupal. 


\section{Discusión y conclusiones}

En relación con el objetivo propuesto en el presente estudio, tras haberse implementado la metodología de indagación guiada en prácticas de laboratorio de física, se evidenció que hubo un progreso significativo en la apropiación de las competencias científicas propuestas al interior de la investigación en los estudiantes de educación media de las tres instituciones educativas objeto de estudio. Esto se comprobó al darse un aumento significativo en los resultados generales de la prueba de salida en donde los estudiantes de grado décimo del colegio Hunzá presentaron un aumento hasta del $15 \%$ y los de grado once del $18 \%$. Asimismo, los estudiantes de grado décimo del colegio San Simón presentaron un aumento del $13 \%$ y los de grado undécimo del 11\%. Los estudiantes de grado décimo y undécimo del colegio Abraham Lincoln, aunque presentaron un aumento de menor proporción, $9 \%$ y $5.9 \%$ respectivamente, siguieron presentando los desempeños más altos, lo cual, se debe en gran medida a la formación en competencias que presentan los estudiantes desde grados inferiores.

En cuanto a los resultados obtenidos por los estudiantes en cada competencia en la prueba de salida, se concluye también que, en las tres instituciones educativas la competencias que más alto resultado obtuvo fue la competencia $U C \mathrm{y}$, de manera preocupante, la competencia que menos resultado obtuvo fue la de $P R C$, lo que implica que al interior de las clases de ciencias debe darse un trabajo más exhaustivo y riguroso para motivar el desarrollo de procesos cognitivos en los estudiantes, fortaleciendo procesos mentales como la seriación, la discriminación, la clasificación, el análisis y la síntesis, hallazgos que van en la línea del estudio de Torres, Mora, Garzón y Ceballos (2013). Por el contrario, estos resultados, no se muestran acordes con los avances obtenidos por los estudiantes al interactuar con las prácticas de laboratorio.

Sobre el trabajo experimental en el aula, puede concluirse que la metodología implementada propició adecuar prácticas que resultaron llamativas e interesantes, tanto para los estudiantes como para los docentes titulares de la asignatura de física en cada uno de los grados en las diferentes instituciones educativas. De esta forma, los estudiantes interactuaron con diferentes tipos de prácticas (virtuales, guiadas, entre otras), diseñadas con toda clase de recursos. Este aspecto avaló la motivación inicial de aplicar la estrategia metodología en diferentes contextos educativos, mostrando resultados que le dan un alcance significativo a la propuesta en el campo de la educación, pues brinda herramientas tanto a docentes como a estudiantes para la apropiación de competencias científicas en las diferentes asignaturas de ciencia en forma dinámica y llamativa bajo diferentes contextos.

De esta forma, se espera que la investigación desarrollada tenga un impacto positivo sobre los docentes de ciencias que exponen que no contar con recursos o con el tiempo necesario constituye en una limitación para la implementación de prácticas experimentales. Así mismo este estudio identifica la necesidad de reflexión docente, mostrando que el desarrollo de competencias científicas no solo debe centrarse en la resolución de problemas de lápiz y papel o la aplicación de pruebas escritas al interior del aula, sino que deben promoverse espacios que denotan un especial interés para los estudiantes como los son los laboratorios (Guerrero, 2011), implementando al interior de ellos, metodologías donde se potencia la curiosidad científica y se apropian las 
habilidades necesarias para desarrollar un pensamiento crítico y razonamiento científico (Torres, 2010).

No obstante lo anterior, a pesar de los excelentes resultados obtenidos en la investigación, encontramos algunas limitaciones en la implementación de la propuesta, siendo destacable: a) La resistencia de algunos rectores y docentes de instituciones educativas a las cuales se les invitó a participar; y, b) el miedo por afrontar nuevas perspectivas en la educación deja a la enseñanza encerrada en modelos tradicionales y cotidianos, desplazando la oportunidad de implementar nuevas alternativas pedagógicas que alimenten y fortalezcan el quehacer didáctico.

Para finalizar, aunque se reconoce que la metodología de indagación es trabajada ampliamente a nivel mundial, en Colombia no hay lineamientos claramente definidos para su implementación, específicamente en prácticas de laboratorio en educación media. De esta forma nuevos interrogantes se abren hacia la posibilidad de transferencia de esta metodología en otros colegios, y hacia la influencia de determinadas variables que pueden mediar en su impacto; como pueden ser, la concienciación de colegios y docentes sobre nuevas metodologías y la experiencia previa en acciones de innovación educativa.

\section{Referencias}

Álvarez-Gayou, J. (2003). Como hacer investigación cualitativa. Fundamentos y metodología. México: Paidós Educador.

Arnal, J., Del Rincón, D., \& Latorre, A. (1992). Investigación educativa. Fundamentos y metodologías. Barcelona: Labor.

Benarroch, A., \& Níñez, G. (2015). Aprendizaje de competencias científicas versus aprendizaje de contenidos específicos. Una propuesta de evaluación. Enseñanza de las ciencias, 33(2), 9-27. doi: 10.5565/rev/ensciencias.1578

Bybee, R. (2004). Scientific Inquiry and Science Teaching. En: Flick, L. y Lederman N. (eds.), Scientific inquiry and nature of science: Implications for teaching, learning, and teacher education. Dordrecht, The Netherlands: Kluwer Academic Publishers,.

Caamaño, A. (2012). ¿Cómo introducir la indagación en el aula? Los trabajos prácticos investigativos. Alambique Didáctica de las ciencias Experimentales(70), 83-91.

Crujeiras, B., \& Jiménez, M. (2015). Análisis de la competencia científica de alumnado de secundaria: respuestas y justificaciones a ítems de PISA. Eureka, 13(2), 385401. Retrieved from https://revistas.uca.es/index.php/eureka/article/view/2931

Daza-Caicedo, S., Guerrero, J., Osorio, C., Hernández, Y., Celis, J., Rozo, C., \& Escobar, M. (2011). Entre datos y relatos: percepciones de jóvenes estudiantes sobre la ciencia. Bogotá: Observatorio Colombiano de Ciencia y Tecnología.

Guerrero, J. (2011). Resultados globales de la encuesta de percepción a jóvenes escolarizados sobre la ciencia, la tecnología, la profesión científica y la enseñanza de las ciencias en Bogotá. En S. Daza-Caicedo, J. Guerrero, C. Osorio, Y. Hernández, J. Celis, C. Rozo, \& M. Escobar, Entre datos y relatos. Percepciones de jóvenes escolarizados sobre la ciencia y la tecnología (págs. 17-74). Bogotá: Observatorio Colombiano de Ciencia y Tecnología. 
Gutiérrez, A. (2008). La evaluación de las competencias científicas en PISA: perfiles en los estudiantes iberoamericanos. Alambique, 57, 23-31. Retrieved from https://didacticaciencias.files.wordpress.com/2010/04/gutierrez_2008.pdf

Högström, P., Ottander, C., \& Benckert, S. (2010). Lab work and learning in secondary school chemistry: the importance of teacher and student interaction. Research in Science Education, 40, 505-523. Retrieved from https://link.springer.com/article/10.1007/s11165-009-9131-3

ICFES. (2017). Saber $11^{\circ}$ 2017-2. Bogotá: Instituto para el Fomento de la Educación Superior.

OCDE. (2006). PISA 2006. Marco de la evaluación. Conocimientos y habilidades en Ciencias, Matemáticas y Lectura. OCDE.

OCDE. (2016). PISA 2015. Resultados Clave. OCDE.

OECD. (2007). PISA 2006 Science Competencies for Tomorrow's World (Vol. 1). OECD Publishing.

Pedrinaci, E., Caamaño, A., Cañal, P., \& de Pro, A. (2012). Once ideas clave. El desarrollo de la competencia científica. Barcelona: Graó.

Rocard, M., Csermely, P., Jorde, D., Lenzen, D., Walwerg-Henriksson, H., \& Hemmo, V. (2008). Enseñanza de las ciencias ahora: una nueva pedagogía para el futuro de Europa. Alambique. Didáctica de las ciencias experimentales(55), 104-120.

Séré, M. G. (2003). La enseñanza en el laboratorio. ¿Qué podemos aprender en términos de conocimiento práctico y de actitudes hacia la ciencia? Enseñanza de las Ciencias, 20(3), 357-368. Retrieved from https://core.ac.uk/download/pdf/38990709.pdf

Sjøberg, S., \& Schereiner, C. (2004). The ROSE project. An overview and key findings. Retrieved from https://roseproject.no/network/countries/norway/eng/norSjoberg-Schreiner-overview-2010.pdf

Torres, Á., Mora, E., Garzón, F., \& Ceballos, N. (2013). Desarrollo de competencias científicas a través de la aplicación de estrategias didácticas alternativas. Un enfoque a través de la enseñanza de las ciencias naturales. Tendencias. Revista de la Facultad de Ciencias Económicas y Administrativas. Universidad de Nariño, XIV(1), 187-215. Retrieved from https://dialnet.unirioja.es/servlet/articulo?codigo $=4453237$

Torres, M. (2010). La enseñanza tradicional de las ciencias versus las nuevas tendencias educativas. Revista electrónica Educare, XIN(1), 131-142. Retrieved from https://dialnet.unirioja.es/servlet/articulo?codigo $=4780946$

Fecha de recepción: 29/10/2019

Fecha de revisión: 28/11/2019

Fecha de aceptación: 27/02/2019 